\title{
ON LINEAR INDEPENDENCE FOR INTEGER TRANSLATES OF A FINITE NUMBER OF FUNCTIONS
}

\author{
RONG-QING JIA and CHARLES A. MICCHELLI
}

(Received 3rd January 1991)

\begin{abstract}
We investigate linear independence of integer translates of a finite number of compactly supported functions in two cases. In the first case there are no restrictions on the coefficients that may occur in dependence relations. In the second case the coefficient sequences are restricted to be in some $l^{p}$ space $(1 \leqq p \leqq \infty)$ and we are interested in bounding their $l^{p}$-norms in terms of the $L^{p}$-norm of the linear combination of integer translates of the basis functions which uses these coefficients. In both cases we give necessary and sufficient conditions for linear independence of integer translates of the basis functions. Our characterization is based on a study of certain systems of linear partial difference and differential equations, which are of independent interest.
\end{abstract}

1980 Mathematics subject classification (1985 Revision): 41A30, 41A63, 15A03, 35E20, 39A70

\section{Introduction}

In this paper, we are concerned with spaces generated by integer translates of a finite number of compactly supported functions. Specifically, given a finite set $\Phi$ of compactly supported functions $\phi_{1}, \ldots, \phi_{n}$ from $\mathbb{R}^{s}$ to $\mathbf{C}$, we are interested in the space

$$
F(\Phi):=F\left(\phi_{1}, \ldots, \phi_{n}\right):=\left\{\sum_{j=1}^{n}\left[\phi_{j}, f_{j}\right]: f_{1}, \ldots, f_{n} \in S\right\}
$$

where $S$ is the linear space of all sequences $f: \mathbb{Z}^{s} \rightarrow \mathbf{C}$, and

$$
[\phi, f]:=\sum_{\alpha \in \mathbf{Z}^{s}} \phi(\cdot-\alpha) f(\alpha)
$$

These spaces have already been a focus of a lively investigation, see the recent monograph [3] and references therein. We only mention here that it was Schoenberg [15] and later Fix and Strang [8] who studied the question when polynomials of some fixed total degree are contained in $F(\Phi)$. This issue is important for constructing finite element approximations.

Motivated by other questions concerning multivariate splines, one is led to consider the problem of algebraic linear independence of integer translates of $\phi_{1}, \ldots, \phi_{n}$. Here "algebraic" means that there are no restrictions on the coefficients that may occur. 
Precisely, the integer translates of $\phi_{1}, \ldots, \phi_{n}$ are said to be (algebraically) linearly independent provided the linear mapping

$$
T:\left(f_{1}, \ldots, f_{n}\right) \mapsto \sum_{j=1}^{n}\left[\phi_{j}, f_{j}\right]
$$

from $S^{n}$ to $F(\Phi)$ is injective. One of the main purposes of this paper is to give a characterization for linear independence in terms of the Fourier-Laplace transforms of $\phi_{1}, \ldots, \phi_{n}$.

We shall investigate the properties of the mapping $T$ when $f_{1}, \ldots, f_{n}$ are restricted to be elements of $l^{p}\left(\mathbb{Z}^{s}\right)$ for some $p, 1 \leqq p \leqq \infty$. If $\phi_{1}, \ldots, \phi_{n}$ are continuous functions of compact support, then one can show that the image of $\left(l^{p}\left(\mathbb{Z}^{s}\right)\right)^{n}$ under the mapping $T$ is a subspace of $L^{p}\left(\mathbb{P}^{s}\right)$, which we shall denote by $F_{p}\left(\phi_{1}, \ldots, \phi_{n}\right)$, or simply $F_{p}(\Phi)$. If $T$ is injective as a mapping from $S^{n}$ to $F(\Phi)$, then the restriction of $T$ to $\left(l^{\infty}\left(\mathbb{Z}^{s}\right)\right)^{n}$ is also injective. It turns out that this latter condition on $T$ is equivalent to the boundedness of $T^{-1}$ as a mapping from $F_{p}(\Phi)$ to $\left(l^{p}\left(\mathbb{Z}^{s}\right)\right)^{n}$ for all $p, 1 \leqq p \leqq \infty$. We shall also give a necessary and sufficient condition in terms of the Fourier transforms of $\phi_{1}, \ldots, \phi_{n}$ for this to happen.

The problem of linear independence of integer translates of basis functions stemmed from some questions about multivariate splines. In [1] de Boor and Höllig considered the linear independence problem for integer translates of a box spline. They gave a necessary condition for integer translates of a box spline to be linearly independent and conjectured that their condition would be also sufficient. Their conjecture was confirmed independently by Dahmen and Micchelli [5], and Jia [10].

The general problem of linear independence of integer translates of a function was studied in [5]. It was realized there that a dependence relation, that is, an element $f \in S$ with $[\phi, f]=0$ could be viewed as a family of difference relations on the element $f$. Thus one was led to focus on the ideal generated by these difference equations, invoke the Hilbert Nullstellensatz and prove that the existence of a nontrivial dependence relation implies the existence of a dependence relation for some exponential element $f_{\theta} \in S$ with $\theta=\left(\theta_{1}, \ldots, \theta_{s}\right) \in(C \backslash\{0\})^{s}$, where $f_{\theta}$ is defined by the rule

$$
f_{\theta}(\alpha):=\theta^{\alpha}:=\theta_{1}^{\alpha_{1}} \ldots \theta_{s}^{\alpha_{s}} \quad \text { for } \alpha=\left(\alpha_{1}, \ldots, \alpha_{s}\right) \in \mathbb{Z}^{s} .
$$

Obviously the elements $f \in S$ such that $[\phi, f]=0$ form a shift-invariant subspace of $S$ closed under pointwise convergence topology. Thus, as observed in [13], the result mentioned above is a consequence of a general principle of Lefranc [12], which say that every nontrivial closed shift-invariant subspace of $S$ contains an exponential element. Moreover, using the Poisson summation formula, Ron [13] put the above result into the following convenient form: The integer translates of a compactly supported distribution $\phi$ on $\mathbb{R}^{s}$ are linearly dependent if and only if there exists some $\xi \in \mathbf{C}^{s}$ such that

$$
\hat{\phi}(\xi+2 \pi \alpha)=0 \quad \text { for all } \alpha \in \mathbb{Z}^{s}
$$


where $\hat{\phi}$ is the Fourier-Laplace transform of $\phi$.

Our characterization of linear independence is based on a study of certain systems of linear partial difference and differential equations, to which Section 2 is devoted. The results of Section 2 essentially depend on an application of Hilbert's Nullstellensatz. In Section 3 these results are employed to characterize linear independence of integer translates of a finite number of compactly supported distributions. The linear space of all possible linear dependence relations among these integer translates is studied in Section 4 along with certain kernel spaces of linear partial difference and differential operators. Finally, Wiener's lemma will be used in Section 5 to give a useful necessary and sufficient condition for $T^{-1}$ to be a bounded operator from $F_{p}(\Phi)$ to $\left(l^{p}\left(\mathbb{Z}^{s}\right)\right)^{n}$.

\section{Linear partial differential and difference equations}

Let $k$ be an algebraically closed field. Denote by $\Pi\left(k^{s}\right)$ the ring of polynomials over $k$ in $s$ variables. Let $S$ be the linear space of all sequences $f: \mathbb{Z}^{s} \rightarrow k$. For example, for $\theta=\left(\theta_{1}, \ldots, \theta_{s}\right) \in(k \backslash\{0\})^{s}$, the sequence given by

$$
\alpha \mapsto \theta^{\alpha}=\theta_{1}^{\alpha_{1}} \ldots \theta_{s}^{\alpha_{s}}, \quad \alpha=\left(\alpha_{1}, \ldots, \alpha_{s}\right) \in \mathbb{Z}^{s},
$$

is an element of $S$, which we shall denote by $f_{\theta}$.

Let $\left\{e_{1}, \ldots, e_{s}\right\}$ be the standard basis for $\mathbb{R}^{s}$. We denote by $\tau_{j}$ the shift operator given by

$$
\tau_{j} f=f\left(\cdot+e_{j}\right), \quad j=1, \ldots, s
$$

For $\beta=\left(\beta_{1}, \ldots, \beta_{s}\right) \in \mathbb{Z}^{s}$, let

$$
\tau^{\beta}:=\tau_{1}^{\beta_{1}} \ldots \tau_{s}^{\beta_{s}}
$$

If $p \in \Pi\left(k^{s}\right), p(x)=\sum_{\beta \geqq 0} a_{\beta} x^{\beta}$, then $p$ induces the linear partial difference operator

$$
p(\tau):=\sum_{\beta \geqq 0} a_{\beta} \tau^{\beta}
$$

It follows immediately that

$$
p(\tau) f_{\theta}=p(\theta) f_{\theta}, \quad \theta \in(k \backslash\{0\})^{s}
$$

Let $P=\left(p_{i j}\right)_{1 \leqq i \leqq m, 1 \leqq j \leqq n}$ be a matrix with all its entries $p_{i j} \in \Pi\left(k^{s}\right)$. For $\theta \in k^{s}$, we denote by $P(\theta)$ the matrix $\left(p_{i j}(\theta)\right)_{1 \leqq i \leqq m, 1 \leqq j \leqq n}$. Consider the system of linear homogeneous partial difference equations for $\left(f_{1}, \ldots, f_{n}\right) \in S^{n}$ :

$$
\sum_{j=1}^{n} p_{i j}(\tau) f_{j}=0, \quad i=1, \ldots, m
$$


All the solutions to this system of equations form a subspace of $S^{n}$ which we shall denote by $\tau(P)$.

Theorem 2.1. Let $P$ be an $m \times n$ matrix whose entries are elements of $\Pi\left(k^{s}\right)$. Then the following conditions are equivalent.

(i) $\tau(P) \neq 0$.

(ii) There exists some $\theta \in(k \backslash\{0\})^{s}$ and $\left(a_{1}, \ldots, a_{n}\right) \in k^{n} \backslash\{0\}$ such that

$$
\left(a_{1} f_{\theta}, \ldots, a_{n} f_{\theta}\right) \in \tau(P) .
$$

(iii) There exists some $\theta \in(k \backslash\{0\})^{s}$ such that the matrix $P(\theta)$ has rank less than $n$.

Proof. First, by (2.1) we have

$$
\sum_{j=1}^{n} p_{i j}(\tau)\left(a_{j} f_{\theta}\right)=\left(\sum_{j=1}^{n} p_{i j}(\theta) a_{j}\right) f_{\theta}
$$

Hence (2.3) is true if and only if

$$
\sum_{j=1}^{n} p_{i j}(\theta) a_{j}=0, \quad i=1, \ldots, m
$$

This proves that (ii) is equivalent to (iii). Since (ii) implies (i) trivially, it remains to prove (i) $\Rightarrow$ (ii). For this purpose, we consider the ideal $J$ generated by the polynomials $p_{11}, \ldots, p_{m 1}$. Let $V(J)$ denote the variety of $J$, i.e.,

$$
V(J):=\left\{z \in k^{s}: p(z)=0 \text { for all } p \in J\right\}
$$

If $V(J) \cap(k \backslash\{0\})^{s}$ is nonempty, then there is some $\theta \in(k \backslash\{0\})^{s}$ such that

$$
p_{11}(\theta)=0, \ldots, p_{m 1}(\theta)=0
$$

It follows that

$$
\left(f_{\theta}, 0, \ldots, 0\right) \in \tau(P)
$$

Thus the theorem is true in this case. If $V(J) \cap(k \backslash\{0\})^{s}$ is empty, then the monomial $r$ given by

$$
r(x):=x_{1} \ldots x_{s}\left(x=\left(x_{1}, \ldots, x_{s}\right) \in k^{s}\right)
$$

vanishes on $V(J)$. Hence, by Hilbert's Nullstellensatz, there exists a positive integer $k$ such that $r^{k} \in J$. Thus we can find polynomials $u_{1}, \ldots, u_{m} \in \Pi\left(k^{s}\right)$ such that 


$$
r^{k}=u_{1} p_{11}+\cdots+u_{m} p_{m 1}
$$

If $n=1$, then $f \in \tau(P)$ implies

$$
p_{11}(\tau) f=\cdots=p_{m 1}(\tau) f=0
$$

This together with (2.4) gives $r^{k}(\tau) f=0$, and hence $f=0$, because $r^{k}(\tau)$ is invertible on $S$ with $\left(\tau_{1} \ldots \tau_{s}\right)^{-k}$ as its inverse. In other words, if $V(J)$ is disjoint from $(k \backslash\{0\})^{s}$, then $\tau(P)$ is trivial. This proves the theorem for $n=1$.

We proceed with the proof by induction on $n$. Let $n>1$ and suppose that the theorem is true for $n-1$. Set

$$
q_{j}:=\sum_{i=1}^{\mathrm{m}} u_{i} p_{i j}, \quad j=1, \ldots, n
$$

Note that $q_{1}=r^{k}$ by (2.4). Let $\left(f_{1}, \ldots, f_{n}\right) \in \tau(P) \backslash\{0\}$. Then

$$
\sum_{j=1}^{n} q_{j}(\tau) f_{j}=\sum_{j=1}^{n} \sum_{i=1}^{m} u_{i} p_{i j}(\tau) f_{j}=0
$$

In particular, $\left(f_{2}, \ldots, f_{n}\right) \neq 0$, for otherwise $f_{1}$ would also be zero by (2.5). Moreover, by (2.2) and $(2.5),\left(f_{2}, \ldots, f_{n}\right)$ satisfies the following equations:

$$
\sum_{j=2}^{n}\left(q_{1} p_{i j}-p_{i 1} q_{j}\right)(\tau) f_{j}=0, \quad i=1, \ldots, m
$$

Conversely, if $\left(f_{1}, \ldots, f_{n}\right)$ satisifes the equations in (2.5) and (2.6), then it also satisfies the equations in (2.2). Thus the system (2.6) has a nontrivial solution. Therefore by the induction hypthesis one can find some $\theta \in(k \backslash\{0\})^{s}$ and $\left(a_{2}, \ldots, a_{n}\right) \in k^{n-1} \backslash\{0\}$ such that $\left(g_{2}, \ldots, g_{n}\right):=\left(a_{2} f_{\theta}, \ldots, a_{n} f_{\theta}\right)$ is a solution to $(2.6)$. Let

$$
g_{1}:=-\left(\tau_{1} \ldots \tau_{s}\right)^{-k}\left(q_{2}(\tau) g_{2}+\cdots+q_{n}(\tau) g_{n}\right)
$$

Then $g_{1}=a_{1} f_{\theta}$ for some $a_{1} \in k$ by (2.1). Thus 


$$
\left(g_{1}, g_{2}, \ldots, g_{n}\right)=\left(a_{1} f_{\theta}, a_{2} f_{\theta}, \ldots, a_{n} f_{\theta}\right)
$$

satisfies (2.5) and (2.6), hence it is a solution to the system (2.2). This advances the induction step.

Similar results hold for differential operators induced by polynomials. Let $A$ be the linear space of all formal power series over $k$ in $s$ indeterminates $X_{1}, \ldots, X_{s}$. An element of $A$ is of the form $\sum_{\alpha \geqq 0} b_{\alpha} X^{\alpha}$, where $X^{\alpha}:=X_{1}^{\alpha_{1}} \ldots X_{s}^{\alpha_{s}}$ for $\alpha=\left(\alpha_{1}, \ldots, \alpha_{s}\right) \in \mathbb{Z}^{s}$, and the coefficients $b_{\alpha}$ are elements of $k$. For instance, $A$ contains the exponential $e_{\xi}$ for any $\xi=\left(\xi_{1}, \ldots, \xi_{s}\right) \in k^{s}$, which is defined to be the formal power series

$$
\sum_{n=0}^{\infty}(\xi \cdot X)^{n} / n !=\sum_{n=0}^{\infty}\left(\xi_{1} X_{1}+\cdots+\xi_{s} X_{s}\right)^{n} / n !
$$

For $\beta=\left(\beta_{1}, \ldots, \beta_{s}\right) \in \mathbb{Z}^{s}, \beta \geqq 0$, let $D^{\beta}$ be the (formal) differential operator on $A$ given by the rule

$$
D^{\beta}\left(X^{\alpha} / \alpha !\right)=X^{\alpha-\beta} /(\alpha-\beta) !
$$

where we have used the notation $\alpha !:=\alpha_{1} ! \ldots \alpha_{s} !$ and adopted the convention that $X^{\alpha-\beta}=0$ if $\alpha_{j}<\beta_{j}$ for some $j \in\{1, \ldots, s\}$. If $p \in \Pi\left(k^{s}\right), p(x)=\sum_{\beta \geqq 0} a_{\beta} x^{\beta}$, then $p$ induces the linear partial differential operator $p(D):=\sum_{\beta \geq 0} a_{\beta} D^{\beta}$. It follows at once that

$$
p(D)\left(e_{\xi}\right)=p(\xi) e_{\xi}, \quad \xi \in k^{s}
$$

Again, let $P=\left(p_{i j}\right)_{1 \leqq i \leqq m, 1 \leqq j \leqq n ~}$ be a matrix of polynomials. Consider the following system of linear homogeneous partial differential equations for $\left(f_{1}, \ldots, f_{n}\right) \in A^{n}$ :

$$
\sum_{j=1}^{n} p_{i j}(D) f_{j}=0, \quad i=1, \ldots, m
$$

All the solutions to this system of equations form a subspace of $A^{n}$ which we shall denote by $D(P)$.

Theorem 2.2. Let $P$ be an $m \times n$ matrix whose entries are elements of $\Pi\left(k^{s}\right)$. Then the following conditions are equivalent.

(i) $D(P) \neq 0$.

(ii) There exists some $\xi \in k^{s}$ and $\left(a_{1}, \ldots, a_{n}\right) \in k^{n} \backslash\{0\}$ such that

$$
\left(a_{1} e_{\xi}, \ldots, a_{n} e_{\xi}\right) \in D(P) \text {. }
$$

(iii) There exists some $\xi \in k^{s}$ such that the matrix $P(\xi)$ has rank less than $n$. 


\section{Algebraic linear independence}

In this section we let $k$ be the complex field $\mathbf{C}$. Recall that a distribution on $\mathbb{R}^{s}$ is a continuous linear functional on the test function space $\mathscr{D}\left(\mathbb{R}^{s}\right)$ (e.g., see $[14$, Chap. 6]). The space of all distributions on $\mathbb{R}^{s}$ is denoted by $\mathscr{D}^{\prime}\left(\mathbb{R}^{s}\right)$. If $\phi \in \mathscr{D}^{\prime}\left(\mathbb{R}^{s}\right)$ is compactly supported, then the Fourier-Laplace transform of $\phi$, denoted by $\hat{\phi}$, is defined to be the function given by

$$
\xi \mapsto\left\langle\phi, e_{-i \xi}\right\rangle, \quad \xi \in C^{s},
$$

where $i$ denotes the imaginary unit. It is well known that $\hat{\phi}$ is an entire function on $\mathbf{C}^{s}$ and its restriction to $\mathbb{R}^{s}$ is the Fourier transform of $\phi$ (see [14, Theorem 7.23]).

For a compactly supported distribution $\phi$ on $\mathbb{R}^{s}$ and a sequence $f \in S$, the bilinear sum of $\phi$ and $f$, denoted by $[\phi, f]$, is the distribution defined by (1.2). Let $\Phi=\left\{\phi_{1}, \ldots, \phi_{n}\right\}$ be a finite set of compactly supported distributions on $\mathbb{R}^{s}$. Recall that the linear mapping $T$ defined in (1.3) maps $S^{n}$ onto $F(\Phi)$, where $F(\Phi)$ is as given in (1.1). We denote by $K(\Phi)=K\left(\phi_{1}, \ldots, \phi_{n}\right)$ the kernel of $T$; that is,

$$
K(\Phi):=\left\{\left(f_{1}, \ldots, f_{n}\right) \in S^{n}: \sum_{j=1}^{n}\left[\phi_{j}, f_{j}\right]=0\right\}
$$

If $K(\Phi)$ is trivial, i.e., $K(\Phi)=0$, then the integer translates of $\phi_{1}, \ldots, \phi_{n}$ are said to be (algebraically) linearly independent. The purpose of this section is to give a characterization for $K(\Phi)$ to be trivial.

Lemma 3.1. There exists a matrix $P$ with $n$ columns whose entries are elements of $\Pi\left(C^{s}\right)$ such that $K(\Phi)=\tau(P)$.

Proof. Let $G$ be the open cube $(-1,1)^{s}$. Since $\mathbb{R}^{s}$ is the union of $G+\beta, \beta \in \mathbb{Z}^{s}$, we see that $\left(f_{1}, \ldots, f_{n}\right) \in K(\Phi)$ if and only if

$$
\left.\tau^{\beta}\left(\sum_{j=1}^{n}\left[\phi_{j}, f_{j}\right]\right)\right|_{G}=0 \text { for all } \beta \in \mathbb{Z}^{s}
$$

But $\tau^{\beta}[\phi, f]=\left[\phi, \tau^{\beta} f\right]$; hence $\left(f_{1}, \ldots, f_{n}\right) \in K(\Phi)$ if and only if

$$
\left.\sum_{j=1}^{n} \sum_{a \in Z^{s}}\left(\tau^{\beta} f_{j}\right)(\alpha) \phi_{j}(\cdot-\alpha)\right|_{G}=0 \quad \text { for all } \beta \in \mathbb{Z}^{s}
$$

Since $\phi_{1}, \ldots, \phi_{n}$ are compactly supported, there exists a positive integer $N$ such that

$$
|\alpha|>\left.N \Rightarrow \phi_{j}(\cdot-\alpha)\right|_{G}=0 \text { for all } j=1, \ldots, n,
$$

where $|\alpha|:=\max \left\{\left|\alpha_{1}\right|, \ldots,\left|\alpha_{s}\right|\right\}$ for $\alpha=\left(\alpha_{1}, \ldots, \alpha_{s}\right) \in \mathbb{Z}^{s}$. This shows that the restriction of 
the linear space $F(\Phi)$ to $G$ is finite dimensional. Choose a basis $\psi_{1}, \ldots, \psi_{m}$ for it. For $j=1, \ldots, n$ and $\alpha \in \mathbb{Z}^{s},\left.\phi_{j}(\cdot-\alpha)\right|_{G}$ can be uniquely represented as follows:

$$
\left.\phi_{j}(\cdot-\alpha)\right|_{G}=\sum_{i=1}^{m} a_{i j}(\alpha) \psi_{i}
$$

where the coefficients $a_{i j}(\alpha) \in \mathrm{C}$ and are zero for $|\alpha|>N$. In terms of (3.3), (3.1) is equivalent to

$$
\sum_{i=1}^{m}\left(\sum_{j=1}^{n} \sum_{|\alpha| \leqq N} a_{i j}(\alpha)\left(\tau^{\beta} f_{j}\right)(\alpha)\right) \psi_{i}=0 \quad \text { for all } \beta \in \mathbb{Z}^{s}
$$

Note that $\tau^{\beta} f(\alpha)=f(\alpha+\beta)=\tau^{\alpha} f(\beta)$. Since $\psi_{1}, \ldots, \psi_{m}$ are linearly independent, (3.4) is equivalent to

$$
\sum_{j=1}^{n}\left(\sum_{|\alpha| \leqq N} a_{i j}(\alpha) \tau^{\alpha}\right) f_{j}=0, \quad i=1, \ldots, m
$$

Let $\gamma$ be the $s$-vector $(N, \ldots, N)$ and define $p_{i j}$ to be the polynomial given by

$$
p_{i j}(x):=\sum_{|\alpha| \leqq N} a_{i j}(\alpha) x^{\alpha+\gamma}, \quad i=1, \ldots, m, j=1, \ldots, n .
$$

Then $K(\Phi)=\tau(P)$ for the matrix $P:=\left(p_{i j}\right)_{1 \leqq i \leqq m, 1 \leqq j \leqq n}$.

The following lemma is a consequence of the Poisson summation formula (see [13, Lemma 2.1]).

Lemma 3.2. Let $\phi$ be a compactly supported distribution on $\mathbb{R}^{s}$. Then for a given $\xi \in \mathbf{C}^{s}$, the sequence $\left(e^{i \xi \cdot \alpha}\right)_{\alpha \in \mathbf{Z}^{s}}$ lies in $K(\phi)$ if and only if

$$
\hat{\phi}(\xi+2 \pi \alpha)=0 \quad \text { for all } \alpha \in \mathbb{Z}^{s}
$$

Theorem 3.3 Let $\phi_{1}, \ldots, \phi_{n}$ be compactly supported distributions on $\mathbb{R}^{s}$. Then the following conditions are equivalent.

(i) $K\left(\phi_{1}, \ldots, \phi_{n}\right) \neq 0$.

(ii) There exists some $\theta \in(\mathbf{C} \backslash\{0\})^{s}$ and $\left(a_{1}, \ldots, a_{n}\right) \in \mathbf{C}^{n} \backslash\{0\}$ such that

$$
\left(a_{1} f_{\theta}, \ldots, a_{n} f_{\theta}\right) \in K\left(\phi_{1}, \ldots, \phi_{n}\right)
$$

(iii) There exists some $\xi \in \mathrm{C}^{s}$ such that the sequences 


$$
\left(\hat{\phi}_{j}(\xi+2 \pi \alpha)\right)_{\alpha \in Z^{s}}, \quad j=1, \ldots, n
$$

are linearly dependent.

Proof. By Lemma 3.1., $K\left(\phi_{1}, \ldots, \phi_{n}\right)=\tau(P)$ for some matrix $P$ of polynomials; hence the equivalence between (i) and (ii) follows from Theorem 2.1.

Suppose (3.6) is true. Choose $\xi \in \mathbf{C}^{s}$ so that $e^{i \xi}=\theta$, and set

$$
\phi:=\sum_{j=1}^{n} a_{j} \phi_{j}
$$

Then (3.6) and (3.7) imply

$$
\sum_{\alpha \in \mathbf{Z}^{s}} \theta^{\alpha} \phi(\cdot-\alpha)=\sum_{j=1}^{n} \sum_{\alpha \in \mathbf{Z}^{s}} a_{j} \theta^{\alpha} \phi_{j}(\cdot-\alpha)=0
$$

In other words, $f_{\theta} \in K(\phi)$; hence by Lemma 3.2, (3.5) is valid. It follows from (3.5) and (3.7) that

$$
\sum_{j=1}^{n} a_{j} \hat{\phi}_{j}(\xi+2 \pi \alpha)=0 \quad \text { for all } \alpha \in \mathbb{Z}^{s}
$$

Since $\left(a_{1}, \ldots, a_{n}\right) \neq 0$, this proves that (ii) implies (iii).

Finally, suppose (iii) holds. Then there exists some $\left(a_{1}, \ldots, a_{n}\right) \in \mathbf{C}^{n} \backslash\{0\}$ such that (3.8) is true. With $\theta=e^{i \xi}$ and $\phi$ given by (3.7), we obtain (3.5); hence $f_{\theta} \in K(\phi)$, and then (3.6) follows.

\section{Kernel spaces}

Let $P=\left(p_{i j}\right)_{1 \leqq i \leqq m, 1 \leqq j \leqq n}$ be an $m \times n$ matrix with all its entries $p_{i j} \in \Pi\left(k^{s}\right)$. The kernel spaces $D(P)$ and $\tau(P)$ are of independent interest. Some special cases of these kernel spaces in connection with box splines have been studied in [1] and [6]. When $n=1$, [7] gave necessary and sufficient conditions for $D(P)$ or $\tau(P)$ to be finite dimensional, see also [2], [4] and [11]. In this section we investigate the kernel spaces $D(P)$ and $\tau(P)$ in the general case $(n>1)$ and then apply the results to the study of linear dependence relations among integer translates of a finite number of functions.

Theorem 4.1. Let $P$ be an $m \times n$ matrix whose entries are elements of $\Pi\left(k^{s}\right)$. Then the linear space $\tau(P)$ is finite dimensional if and only if the set

$$
\Theta(P):=\left\{\theta \in(k \backslash\{0\})^{s}: \operatorname{rank}(P(\theta))<n\right\}
$$

is finite, and in this case every element $f=\left(f_{1}, \ldots, f_{n}\right) \in \tau(P)$ has the form 


$$
f_{j}(\alpha)=\sum_{\theta \in \boldsymbol{\Theta}(P)} \theta^{\alpha} q_{j, \theta}(\alpha), \quad \alpha \in \mathbb{Z}^{s},
$$

where $q_{j, \theta} \in \Pi\left(k^{s}\right), j=1, \ldots, n$ and $\theta \in \Theta(P)$.

Proof. If $\theta \in \Theta(P)$, then $\operatorname{rank}(P(\theta))<n$; hence there exists $\left(a_{1, \theta}, \ldots, a_{n, \theta}\right) \in k^{n} \backslash\{0\}$ such that

$$
\sum_{j=1}^{n} p_{i j}(\theta) a_{j, \theta}=0, \quad i=1, \ldots, m
$$

By (2.1) it follows that

$$
\left(a_{1, \theta} f_{\theta}, \ldots, a_{n, \theta} f_{\theta}\right) \in \tau(P)
$$

Observe that the set of elements

$$
\left\{\left(a_{1, \theta} f_{\theta}, \ldots, a_{n, \theta} f_{\theta}\right): \theta \in \Theta(P)\right\}
$$

is linearly independent. Thus if $\tau(P)$ is finite dimensional, then $\Theta(P)$ must be a finite set.

Conversely, suppose that $\Theta(P)$ is a finite set. We wish to show that the dimension of $\tau(P)$ is finite and every element $\left(f_{1}, \ldots, f_{n}\right)$ of $\tau(P)$ has the form (4.1). The proof proceeds with induction on $n$. The case $n=1$ was proved in [7, Proposition 2.2] and [11, Theorem 4.1]. Let $n>1$. For $l=1, \ldots, m$, let $q_{i j}^{l} \in \Pi\left(k^{s}\right)$ be given by

$$
q_{i j}^{l}:=\left|\begin{array}{ll}
p_{l 1} & p_{l j} \\
p_{i 1} & p_{i j}
\end{array}\right|, \quad i=1, \ldots, m ; j=2, \ldots, n,
$$

and form the matrix

$$
Q^{l}=\left(q_{i j}^{l}\right)_{1 \leqq i \leqq m, 2 \leqq j \leqq n}
$$

Note that if $p_{l 1}(\theta) \neq 0$, then

$$
\operatorname{rank}(P(\theta))=\operatorname{rank}\left(Q^{l}(\theta)\right)+1
$$

Form the matrix

$$
Q:=\left[\begin{array}{c}
Q^{1} \\
\vdots \\
Q^{n}
\end{array}\right]
$$

and consider the linear space 


$$
\tau(Q):=\left\{\left(f_{2}, \ldots, f_{n}\right) \in S^{n-1}: \sum_{j=2}^{n} q_{i j}^{l}(\tau) f_{j}=0, i, l=1, \ldots, m ; j=2, \ldots, n\right\}
$$

From the construction of $Q$ we see that

$$
\left(f_{1}, f_{2}, \ldots, f_{n}\right) \in \tau(P) \Rightarrow\left(f_{2}, \ldots, f_{n}\right) \in \tau(Q) .
$$

Let

$$
\Theta(Q):=\left\{\theta \in(k \backslash\{0\})^{s}: \operatorname{rank}(Q(\theta))<n-1\right\}
$$

We claim that

$$
\Theta(Q) \subseteq \Theta(P)
$$

Suppose $\theta \notin \Theta(P)$. Then $\operatorname{rank}(P(\theta))=n$. Moreover, $p_{l 1}(\theta) \neq 0$ for some $l \in\{1, \ldots, m\}$, for otherwise $p_{l 1}(\theta)=0$ for all $l \in\{1, \ldots, m\}$ would imply $\theta \in \Theta(P)$. It follows that

$$
\operatorname{rank}(Q(\theta)) \geqq \operatorname{rank}\left(Q^{l}(\theta)\right)=\operatorname{rank}(P(\theta))-1=n-1 .
$$

Hence $\theta \notin \Theta(Q)$. This proves (4.3). Thus $\Theta(Q)$ is a finite set; hence by the induction hypothesis, $\tau(Q)$ is finite dimensional. Let $\pi_{j}(j \in\{1, \ldots, n\})$ be the projection operator from $S^{n}$ to $S$ given by

$$
\left(f_{1}, \ldots, f_{n}\right) \mapsto f_{j}
$$

We have proved that $\pi_{j}(\tau(P))$ is finite dimensional for $j=2, \ldots, n$. The same is true for $j=1$. We conclude therefore that $\tau(P)$ is finite dimensional. Moreover, by (4.2) and the induction hypothesis, $f_{j}$ has the form $(4.1)$ for $j=2, \ldots, n$. The same is true for $j=1$. This completes the induction step.

Similar results hold for the kernel space $D(P)$.

Theorem 4.2. Let $P$ be an $m \times n$ matrix whose entries are elements of $\Pi\left(k^{s}\right)$. Then the linear space $D(P)$ is finite dimensional if and only if the set

$$
\Xi(P):=\left\{\zeta \in k^{s}: \operatorname{rank}(P(\xi))<n\right\}
$$

is finite, and in this case every element $\left(f_{1}, \ldots, f_{n}\right) \in D(P)$ has the form

$$
f_{j}=\sum_{\xi \in \Xi(P)} e_{\xi} q_{j, \xi}
$$

where $q_{j, \xi} \in \Pi\left(k^{s}\right), j=1, \ldots, n$ and $\xi \in \Xi(P)$. 
In the rest of this section we assume that $k$ is the complex field $\mathbf{C}$ and $\Phi=\left\{\phi_{1}, \ldots, \phi_{n}\right\}$ is a finite set of compactly supported distributions on $\mathbb{R}^{s}$. By virtue of Lemma 3.1, the above results can be applied to $K(\Phi)$. We denote by $N(\Phi)$ the set of all $\xi \in \mathrm{C}^{s}$ satisfying $\operatorname{Re}(\xi) \in[0,2 \pi)^{s}$ and the condition that the sequences

$$
\left(\hat{\phi}_{j}(\xi+2 \pi \alpha)\right)_{\alpha \in \mathbb{Z}^{s}}, \quad j=1, \ldots, n,
$$

are linearly dependent.

Theorem 4.3. The kernel space $K(\Phi)$ is finite dimensional if and only if $N(\Phi)$ is a finite set, and in this case any linear dependence relation $\left(f_{1}, \ldots, f_{n}\right) \in K(\Phi)$ has the form

$$
f_{j}(\alpha)=\sum_{\xi \in N(\Phi)} e^{i \xi \cdot \alpha} q_{j, \xi}(\alpha), \quad \alpha \in \mathbb{Z}^{s}
$$

where $q_{j, \xi} \in \Pi\left(\mathbf{C}^{s}\right), j=1, \ldots, n, \xi \in N(\Phi)$.

Proof. The case $n=1$ was proved in [4, Theorem 2.1]. The general case $(n>1)$ follows from Lemmas 3.1 and 3.2 and Theorem 4.1 .

\section{Linear independence on $\boldsymbol{l}^{p}\left(\mathbb{Z}^{s}\right)$}

Let us assume now that $\phi_{1}, \ldots, \phi_{n} \in C_{0}\left(\mathbb{R}^{s}\right)$, the space of complex-valued continuous functions on $\mathbb{R}^{s}$ having compact support. For every $p, 1 \leqq p \leqq \infty$, and $\left(f_{1}, \ldots, f_{n}\right) \in S^{n}$ we define

$$
\left\|\left(f_{1}, \ldots, f_{n}\right)\right\|_{p}:=\max _{1 \leqq j \leqq n}\left\|f_{j}\right\|_{p}
$$

where $\left\|f_{j}\right\|_{p}$ denotes the standard $l^{p}\left(\mathbb{Z}^{s}\right)$ norm of $f_{j} ;$ the $L^{p}\left(\mathbb{R}^{s}\right)$ norm of functions is denoted similarly.

Let $T$ be the mapping as defined in (1.3). In the previous sections we were concerned with the algebraic aspects of $T$. In this section we shall investigate the properties of the operator $T$ when $f_{1}, \ldots, f_{n}$ are restricted to be elements of $l^{p}\left(\mathbb{Z}^{s}\right), 1 \leqq p \leqq \infty$.

First, we show that $T$ maps $\left(l^{p}\left(\mathbb{Z}^{s}\right)\right)^{n}$ into $L^{p}\left(\mathbb{R}^{s}\right)$. For this purpose we make use of the generalized Young inequality (see [9, pp. 13-14]) to estimate the $L^{p}$-norm of $[\phi, f]$, where $\phi \in C_{0}\left(\mathbb{R}^{s}\right)$ and $f \in l^{p}\left(\mathbb{Z}^{s}\right)$. We view $\mathbb{Z}^{s}$ as a measure space by defining the measure of any subset of $\mathbb{Z}^{s}$ to be its cardinality. If $\alpha$ is fixed, we have

$$
\int_{\mathbf{R}^{s}}|\phi(x-\alpha)| d x=\|\phi\|_{1}
$$

if $x$ is fixed, then 


$$
\sum_{\alpha \in Z^{s}}|\phi(x-\alpha)| \leqq \max _{x \in[0,1]^{s}} \sum_{\alpha \in Z^{s}}|\phi(x-\alpha)|=:|\phi|_{\infty}
$$

which is finite because $\phi \in C_{0}\left(\mathbb{R}^{s}\right)$. Evidently, $\|\phi\|_{1} \leqq|\phi|_{\infty}$. Thus by the generalized Young inequality,

$$
\|[\phi, f]\|_{p} \leqq|\phi|_{\infty}\|f\|_{p}, \quad 1 \leqq p \leqq \infty
$$

It follows that

$$
\left\|T\left(f_{1}, \ldots, f_{n}\right)\right\|_{p} \leqq \sum_{j=1}^{n}\left\|\left[\phi_{j}, f_{j}\right]\right\|_{p} \leqq \sum_{j=1}^{n}\left|\phi_{j}\right|_{\infty}\left\|f_{j}\right\|_{p}
$$

In other words, $T$ is a bounded operator from $\left(l^{p}\left(\mathbb{Z}^{s}\right)\right)^{n}$ to $L^{p}\left(\mathbb{R}^{s}\right)$. Also, from the definition of the norm $|\cdot|_{\infty}$ we find that

$$
|[\phi, f]|_{\infty} \leqq|\phi|_{\infty}\|f\|_{1}, \quad \phi \in C_{0}\left(\mathbb{R}^{s}\right), f \in l^{1}\left(\mathbb{Z}^{s}\right) .
$$

Let $\phi, \psi \in C_{0}\left(\mathbb{R}^{s}\right)$ and let $b$ be the sequence on $\mathbb{Z}^{s}$ given by

$$
b(\alpha):=\int_{\mathbf{R}^{s}} \phi(\alpha-x) \psi(x) d x, \quad \alpha \in \mathbb{Z}^{s}
$$

Again, we can invoke the generalized Young inequality to obtain

$$
\|b\|_{p} \leqq|\phi|_{\infty}\|\psi\|_{p}, \quad 1 \leqq p \leqq \infty
$$

The following result has found important applications in the study of wavelets (see R. Q. Jia and C. A. Micchelli, Using the refinement equation for the construction of pre-wavelets II: Powers of two, in Curves and Surfaces, P. J. Laurent, A. Le Méhauté and L. L. Schumaker (eds.), Academic Press, New York, 1991, pp. 209-246).

Theorem 5.1. Let $\phi_{1}, \ldots, \phi_{n} \in C_{0}\left(\mathbb{R}^{s}\right)$. Then the following conditions are equivalent.

(i) There is no $\left(f_{1}, \ldots, f_{n}\right) \in\left(l^{\infty}\left(\mathbb{Z}^{s}\right)\right)^{n} \backslash\{0\}$ such that $\sum_{j=1}^{n}\left[\phi_{j}, f_{j}\right]=0$.

(ii) There exists no $\xi \in \mathbb{R}^{s}$ such that the sequences

$$
\left(\hat{\phi}_{j}(\xi+2 \pi \alpha)\right)_{a \in \mathbf{Z}^{\mathbf{s}}}, \quad j=1, \ldots, n,
$$

are linearly dependent.

(iii) There exists a constant $\delta>0$ such that for all $p, 1 \leqq p \leqq \infty$, and any $f_{1}, \ldots, f_{n} \in l^{p}\left(\mathbb{Z}^{s}\right)$

$$
\delta\left\|\left(f_{1}, \ldots, f_{n}\right)\right\|_{p} \leqq\left\|\sum_{j=1}^{n}\left[\phi_{j}, f_{j}\right]\right\|_{p}
$$


Proof. Suppose the sequences in (5.4) are linearly dependent for some $\xi \in \mathbb{R}^{s}$. Then there exists a vector $\left(a_{1}, \ldots, a_{n}\right) \in \mathbf{C}^{n} \backslash\{0\}$ such that

$$
\sum_{j=1}^{n} a_{j} \hat{\phi}_{j}(\xi+2 \pi \alpha)=0 \text { for all } \alpha \in \mathbb{Z}^{s}
$$

By Lemma 3.2, it follows that

$$
\sum_{\alpha \in Z^{*}} \sum_{j=1}^{n} \phi_{j}(\cdot-\alpha) a_{j} e^{i \xi \cdot \alpha}=0
$$

which contradicts the condition (i). Thus (i) implies (ii). Also, (iii) implies (i) trivially. It remains to prove (ii) $\Rightarrow$ (iii).

For $j, k=1, \ldots, n$, let

$$
b_{j k}(\alpha):=\int_{\mathbb{R}^{s}} \phi_{j}(x+\alpha) \phi_{k}(x) d x, \quad \alpha \in \mathbb{Z}^{s}
$$

where $\bar{\phi}$ denotes the complex conjugate of $\phi$, and let

$$
A_{j k}(\xi):=\sum_{\alpha \in Z^{s}} b_{j k}(\alpha) e^{-i \alpha \cdot \xi}, \quad \xi \in \mathbb{R}^{s}
$$

We claim that the Gram matrix

$$
A(\xi):=\left(A_{j k}(\xi)\right)_{1 \leqq j, k \leqq n}
$$

is positive definite for all $\xi \in \mathbb{R}^{s}$. Evidently, $A(\xi)$ is hermitian. To prove that $A(\xi)$ is positive definite, it suffices to show that

$$
I(\xi):=\sum_{j, k=1}^{n} a_{j} A_{j k}(\xi) \bar{a}_{k}>0
$$

for all $\left(a_{1}, \ldots, a_{n}\right) \in \mathbf{C}^{n} \backslash\{0\}$. It follows from (5.6) and (5.7) that

$$
\begin{aligned}
I(\xi)= & \sum_{\alpha \in \mathbf{Z}^{s}} \sum_{j, k=1}^{n} \int_{\mathbf{R}^{s}} a_{j} \phi_{j}(x+\alpha) \bar{a}_{k} \bar{\phi}_{k}(x) e^{-i \alpha \cdot \xi} d x \\
= & \sum_{\alpha \in \mathbf{Z}^{*}} \int_{\mathbf{R}^{s}} \phi(x+\alpha) \bar{\phi}(x) e^{-i \alpha \cdot \xi} d x,
\end{aligned}
$$

where $\phi:=\sum_{j=1}^{n} a_{j} \phi_{j}$. Note that $\mathbb{R}^{s}$ is the disjoint union of $\beta+[0,1)^{s}, \beta \in \mathbb{Z}^{s}$; hence (5.9) implies 


$$
I(\xi)=\sum_{\alpha, \beta \in \mathbf{Z}^{s}} \int_{[0,1)^{s}} \phi(x+\alpha+\beta) \phi(x+\beta) e^{-i \alpha \cdot \xi} d x=\int_{[0,1)^{s}}|h(x)|^{2} d x
$$

where

$$
h(x):=\sum_{\alpha \in Z^{s}} \phi(x+\alpha) e^{-i \alpha \cdot \xi}
$$

If the condtion (ii) is satisfied, then for any given $\xi \in \mathbb{R}^{s}$ the continuous function $h$ is not identically zero by Lemma 3.2. This verifies (5.8).

Let $A_{j k}^{-1}$ denote the $(j, k)$ th entry of the inverse matrix $(A(\xi))^{-1}$. Every entry $A_{j k}$ is a trigonometric polynomial in $\xi$ by (5.7), so is $\operatorname{det} A$. By Cramer's rule, every $A_{j k}^{-1}$ is a quotient of two trigonometric polynomials for which the denominator never vanishes. By Wiener's lemma (e.g., see $\left[14\right.$, p. 266]), each $A_{j k}^{-1}$ can be expanded into a trigonometric series

$$
A_{j k}^{-1}(\xi)=\sum_{\alpha \in Z^{s}} g_{j k}(\alpha) e^{-i \alpha \cdot \xi}, \quad \xi \in \mathbb{R}^{s}
$$

such that the coefficient sequence $g_{j k} \in l^{1}\left(\mathbb{Z}^{s}\right)$. Define

$$
\gamma_{l}:=\sum_{m=1}^{n}\left[\phi_{m}, g_{l m}\right], \quad l=1, \ldots, n
$$

Fix $k$ and $l$ for the moment. Let

$$
c(\beta):=\left\langle\gamma_{l}, \phi_{k}(\cdot-\beta)\right\rangle:=\int_{\mathbf{R}^{s}} \gamma_{l}(x) \Phi_{k}(x-\beta) d x, \quad \beta \in \mathbb{Z}^{s} .
$$

By (5.6) and (5.10) we have

$$
\begin{aligned}
c(\beta) & =\sum_{m=1}^{n} \sum_{\alpha \in \mathbf{Z}^{s}} \int_{\mathbf{R}^{s}} \phi_{m}(x-\alpha) g_{l m}(\alpha) \phi_{k}(x-\beta) d x \\
& =\sum_{m=1}^{n} \sum_{\alpha \in \mathbf{Z}^{s}} b_{m k}(\beta-\alpha) g_{l m}(\alpha) .
\end{aligned}
$$

It follows that for all $\xi \in \mathbb{R}^{s}$,

$$
\begin{aligned}
\sum_{\beta \in Z^{s}} c(\beta) e^{-i \beta \cdot \xi} & =\sum_{m=1}^{n} \sum_{\alpha, \beta \in Z^{s}} b_{m k}(\beta-\alpha) e^{-i(\beta-\alpha) \cdot \xi} g_{l m}(\alpha) e^{-i \alpha \cdot \xi} \\
& =\sum_{m=1}^{n} A_{m k}(\xi) A_{l m}^{-1}(\xi)=\delta_{k l}
\end{aligned}
$$


where $\delta_{k l}$ is the Kronecker symbol. This shows that

$$
\left\langle\phi_{k}(\cdot-\beta), \gamma_{l}(\cdot-\alpha)\right\rangle=\left\langle\phi_{k}(\cdot-\beta+\alpha), \gamma_{l}\right\rangle=\delta_{\alpha \beta} \delta_{k l}
$$

Thus for the function

$$
\psi:=\sum_{j=1}^{n}\left[\phi_{j}, f_{j}\right]
$$

we obtain the equation

$$
f_{j}(\alpha)=\left\langle\psi, \gamma_{j}(\cdot-\alpha)\right\rangle, \quad \alpha \in \mathbb{Z}^{s}
$$

This in connection with (5.2), (5.3) and (5.10) implies that for $1 \leqq p \leqq \infty$,

$$
\left\|f_{j}\right\|_{p} \leqq\|\psi\|_{p}\left|\gamma_{j}\right|_{\infty} \leqq\|\psi\|_{p} \sum_{m=1}^{n}\left|\phi_{m}\right|_{\infty}\left\|g_{j m}\right\|_{1}, \quad j=1, \ldots, n,
$$

from which (5.5) follows with

$$
\delta^{-1}=\max _{1 \leqq j \leqq n} \sum_{m=1}^{n}\left|\phi_{m}\right|_{\infty}\left\|g_{j m}\right\|_{1}
$$

\section{REFERENCES}

1. C. DE Boor and K. Höllig, B-splines from parallelepipeds, J. Analyse Math. 42 (1982/83), 99-115.

2. C. DE BOOR and A. RoN, On polynomial ideals of finite codimension with applications to box spline theory, Math. Anal. \& Appl. 158 (1991), 168-193.

3. C. K. Chul, Multivariate Splines (CBMS-NSF Regional Conference Series in Applied Mathematics, Vol. 54, SIAM, Philadelphia, 1988).

4. W. Dahmen, R. Q. JiA and C. A. Micchelli, On linear dependence relations for integer translates of compactly supported distributions, Math. Nachr. 151 (1991), 303-310.

5. W. Dahmen and C. A. Micchelli, Translates of multivariate splines, Linear Algebra Appl. 52 (1983), 217-234.

6. W. Dahmen and C. A. Micchelli, On the solution of certain systems of partial difference equations and linear dependence of translates of box splines, Trans. Amer. Math. Soc. 292 (1985), 305-320.

7. W. Dahmen and C. A. Micchelli, Local dimension of piecewise polynomial spaces, syzygies, and solutions of systems of partial differential equations, Math. Nachr. 148 (1990), 117-136.

8. G. Fix and G. Strang, Fourier analysis of the finite element method in Ritz-Galerkin theory, Stud. Appl. Math. 48 (1969), 265-273.

9. G. B. Folland, Introduction to Partial Differential Equations (Princeton University Press, Princeton, New Jersey, 1976). 
10. R. Q. J IA, Linear independence of translates of a box spline, J. Approx. Theory 40 (1984), $158-160$.

11. R. Q. JiA, S. Riemenschneider and Z. W. Shen, Dimension of kernels of linear operators, Amer. J. Math. 114 (1992), 157-184.

12. M. Lefranc, Analyse spectrale sur $Z_{n}$, C.R. Acad. Sci. Paris 246 (1958), 1951-1953.

13. A. Ron, A necessary and sufficient condition for the linear independence of the integer translates of a compactly supported distribution, Constr. Approx. 5 (1989), 297-308.

14. W. Rudin, Functional Analysis (McGraw-Hill Book Company, New York, 1973).

15. I. J. Schoenberg, Contributions to the problem of approximation of equidistant data by analytic functions, Part A, Quart. Appl. Math. 4 (1946), 45-99; Part B, ibid 4 (1946), 112-141.

Department of Mathematics

UNIVERSITY OF ALBERTA

EDMONTON, T6G 2G1

Canada
IBM Research Division

T. J. Watson Research Center

Mathematical Sciences Department

Yorktown Heights, NY 10598, USA 